\title{
Magnetic Monopoles in Spin Ice
}

\author{
C. Castelnovo ${ }^{1}$, R. Moessner ${ }^{1,2}$, and S. L. Sondhi ${ }^{3}$ \\ ${ }^{1}$ Rudolf Peierls Centre for Theoretical Physics, Oxford University, Oxford OX1 3NP, UK \\ ${ }^{2}$ Max-Planck-Institut für Physik komplexer Systeme, 01187 Dresden, Germany and \\ ${ }^{3}$ Department of Physics, Princeton University, Princeton, NJ 08544
}

(Dated: February 12, 2013)

\begin{abstract}
Electrically charged particles, such as the electron, are ubiquitous. By contrast, no elementary particles with a net magnetic charge have ever been observed, despite intensive and prolonged searches ${ }^{1}$. We pursue an alternative strategy, namely that of realising them not as elementary but rather as emergent particles, i.e., as manifestations of the correlations present in a strongly interacting many-body system. The most prominent examples of emergent quasiparticles are the ones with fractional electric charge $e / 3$ in quantum Hall physics ${ }^{2}$. Here we show that magnetic monopoles $d o$ emerge in a class of exotic magnets known collectively as spin ice ${ }^{3,4.5}$ : the dipole moment of the underlying electronic degrees of freedom fractionalises into monopoles. This enables us to account for a mysterious phase transition observed experimentally in spin ice in a magnetic field $\mathrm{d}^{6.7}$, which is a liquid-gas transition of the magnetic monopoles. These monopoles can also be detected by other means, e.g., in an experiment modelled after the celebrated Stanford magnetic monopole search ${ }^{8}$.
\end{abstract}

Spin-ice materials are characterised by the presence of magnetic moments $\vec{\mu}_{i}$ residing on the sites of a pyrochlore lattice (depicted in Fig. 1). These moments are constrained to point along their respective local Ising axes $\hat{e}_{i}$ (the diamond lattice bonds in Fig. 1), and they can be modelled as Ising spins $\vec{\mu}_{i}=\mu S_{i}$, where $S_{i}= \pm 1$ and $\mu=\left|\vec{\mu}_{i}\right|$. For the spin ice compounds discussed here, $\mathrm{Dy}_{2} \mathrm{Ti}_{2} \mathrm{O}_{7}$ and $\mathrm{Ho}_{2} \mathrm{Ti}_{2} \mathrm{O}_{7}$, the magnitude $\mu$ of the magnetic moments equals approximately $10 \mathrm{Bohr}$ magnetons $\left(\mu=10 \mu_{B}\right)$. The thermo-

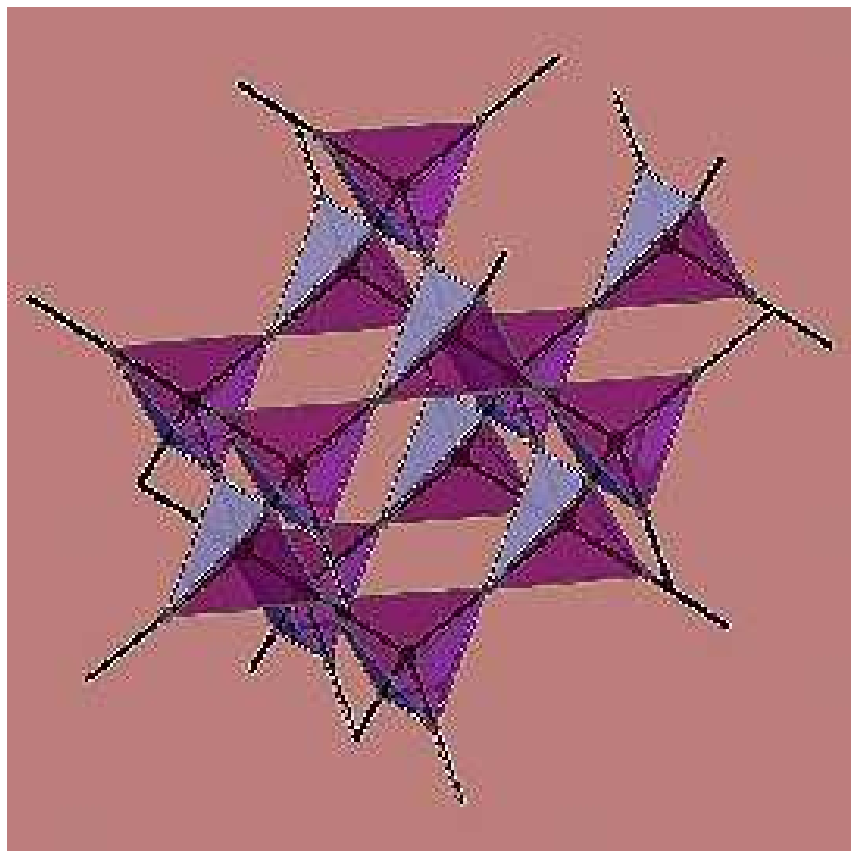

FIG. 1: The pyrochlore and diamond lattices. The magnetic moments in spin ice reside on the sites of the pyrochlore lattice, which consists of corner-sharing tetrahedra. These are at the same time the midpoints of the bonds of the diamond lattice (black) formed by the centres of the tetrahedra. The ratio of the lattice constant of the diamond and pyrochlore lattices is $a_{d} / a=\sqrt{3 / 2}$. The Ising axes are the local [111] directions, which point along the respective diamond lattice bonds. dynamic properties of these compounds are known to be described with good accuracy by an energy term that accounts for the nearest neighbour exchange and the long ranged dipolar interactions ${ }^{4,9,10}$ :

$$
\begin{aligned}
H & =\frac{J}{3} \sum_{\langle i j\rangle} S_{i} S_{j} \\
& +D a^{3} \sum_{(i j)}\left[\frac{\hat{e}_{i} \cdot \hat{e}_{j}}{\left|\mathbf{r}_{i j}\right|^{3}}-\frac{3\left(\hat{e}_{i} \cdot \mathbf{r}_{i j}\right)\left(\hat{e}_{j} \cdot \mathbf{r}_{i j}\right)}{\left|\mathbf{r}_{i j}\right|^{5}}\right] S_{i} S_{j} .
\end{aligned}
$$

The distance between spins is $r_{i j}$, and $a \simeq 3.54 \AA$ is the pyrochlore nearest-neighbour distance. $D=\mu_{0} \mu^{2} /\left(4 \pi a^{3}\right)=$ $1.41 \mathrm{~K}$ is the coupling constant of the dipolar interaction.

Spin ice was identified as a very unusual magnet when it was noted that it does not order to the lowest temperatures, $T$, even though it appeared to have ferromagnetic interactions $\mathrm{s}^{3}$. Indeed, spin ice was found to have a residual entropy at low $T^{5}$, which is well-approximated by the famous Pauling entropy for (water) ice, $S \approx S_{P}=(1 / 2) \log (3 / 2)$ per spin. Pauling's entropy measures the huge ground state degeneracy arising from the so-called ice rules. In the context of spin ice, its observation implies a macroscopically degenerate ground state manifold obeying the "ice rule" that two spins point into each vertex of the diamond lattice, and two out.

We contend that excitations above this ground state manifold, i.e., defects that locally violate the ice rule, are magnetic monopoles with the necessary long distance properties. From the perspective of the seemingly local physics of the ice rule, the emergence of monopoles would at first sight seem rather surprising. To demystify it we will probe deeper into how the long range magnetic interactions contained in Eq. 1 give rise to the ice rule in the first place. We then incorporate insights from recent progress in understanding the entropic physics of spin ice, and the physics of fractionalisation in high dimensions $11,12,13,14,15$, of which our monopoles will prove to be a classical instance.

Consider a modest deformation of Eq. 11, loosely inspired by Nagle's old work ${ }^{16}$ on the 'unit model' description of water 


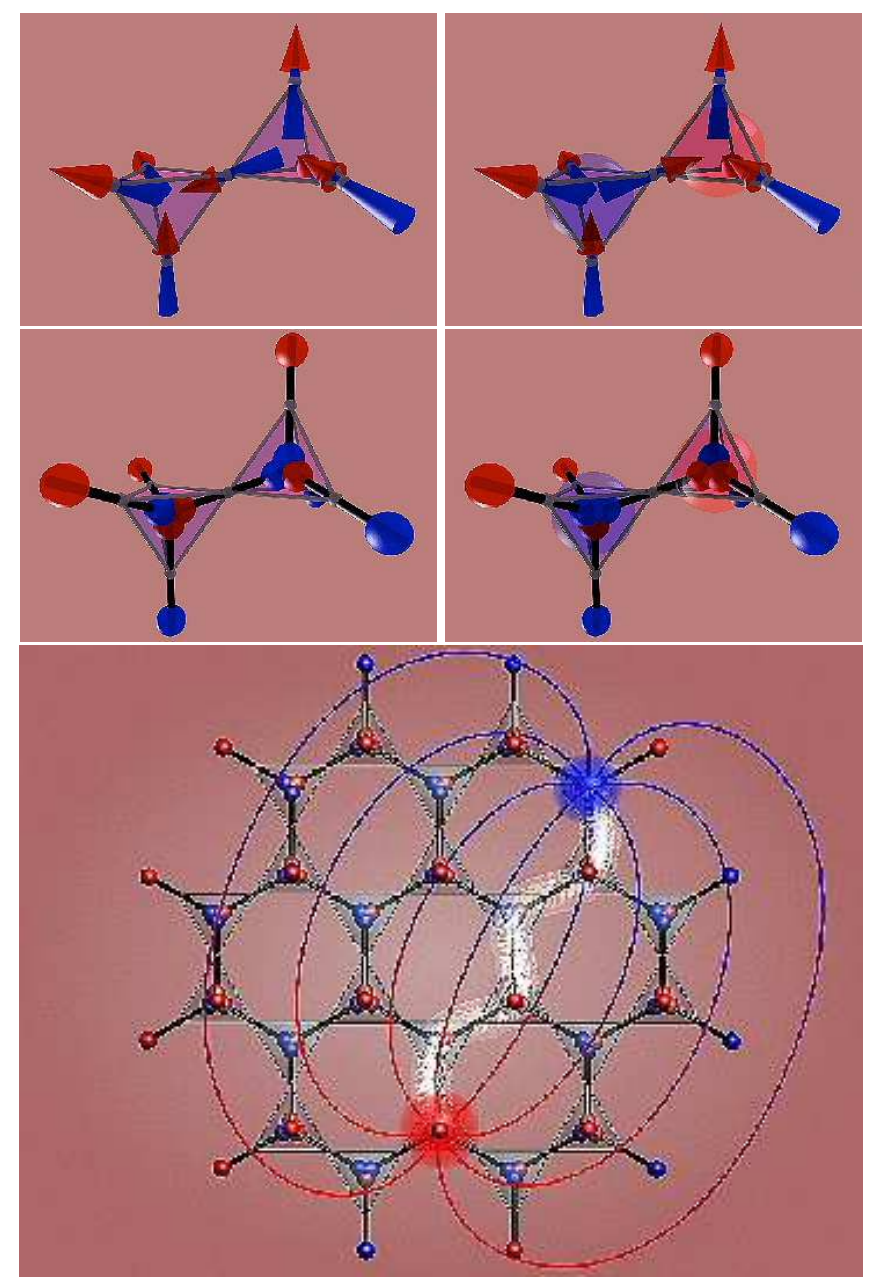

FIG. 2: Mapping from dipoles to dumbbells. The dumbbell picture $(\mathrm{c}, \mathrm{d})$ is obtained by replacing each spin in $(\mathrm{a}, \mathrm{b})$ by a pair of opposite magnetic charges placed on the adjacent sites of the diamond lattice. Left: two neighboring tetrahedra obeying the ice rule, with two spins pointing in and two out, giving zero net charge on each site. Right: Inverting the shared spin generates a pair of magnetic monopoles (diamond sites with net magnetic charge). This configuration has a higher net magnetic moment and it is favoured by an applied magnetic field oriented upward in the figure (corresponding to a [111] direction). Large panel: A pair or separated monopoles (large red and blue spheres). A chain of inverted dipoles ('Dirac string') between them is highlighted, and the magnetic field lines are sketched.

ice: we replace the interaction energy of the magnetic dipoles living on pyrochlore sites by that of dumbbells consisting of equal and opposite magnetic charges that live at the ends of the diamond bonds (see Fig. 2). The two ways of assigning charges on each diamond bond reproduce the two orientations of the original dipole. Demanding that the dipole moment of the spin be reproduced quantitatively fixes the value of the charge at $\pm \mu / a_{d}$, where the diamond lattice bond length $a_{d}=$ $\sqrt{3 / 2} a$.

The energy of a configuration of dipoles is computed as the pairwise interaction energy of magnetic charges, given by the magnetic Coulomb law

$$
V\left(r_{\alpha \beta}\right)= \begin{cases}\frac{\mu_{0}}{4 \pi} \frac{Q_{\alpha} Q_{\beta}}{r_{\alpha \beta}} & \alpha \neq \beta \\ \frac{1}{2} v_{o} Q_{\alpha}^{2} & \alpha=\beta\end{cases}
$$

where $Q_{\alpha}$ denotes the total magnetic charge at site $\alpha$ in the diamond lattice, and $r_{\alpha \beta}$ is the distance between two sites. The finite "self-energy" $v_{0} / 2$ is required to reproduce the net nearest neighbour interaction correctly. This expression - derived in detail in the annexed supplementary notes - is equivalent to the dipolar energy Eq. 1 up to corrections which are small everywhere, and vanish with distance at least as fast as $1 / r^{5}$.

Consider first the ground states of the system. The total energy is minimised if each diamond lattice site is net neutral, i.e., we must orient the dumbbells so that $Q_{\alpha}=0$ on each site. But this is just the above-mentioned ice rule, as illustrated in Fig. 2 Thus, one of the most remarkable features of spin ice pops right out of the dumbbell model: the measured low- $T$ entropy agrees with the Pauling entropy (which follows from the short-distance ice rules), even though the dipolar interactions are long-ranged.

We now turn to the excited states. Naively, the most elementary excitation involves inverting a single dipole / dumbbell to generate a local net dipole moment $2 \mu$. However, this is misleading in a crucial sense. The inverted dumbbell in fact corresponds to two adjacent sites with net magnetic charge

$$
Q_{\alpha}= \pm q_{m}= \pm 2 \mu / a_{d}
$$

a nearest neighbour monopole-antimonopole pair. As shown in Fig. 2, the monopoles can be separated from one another without further violations of local neutrality by flipping a chain of adjacent dumbbells. A pair of monopoles separated by a distance $r$ experiences a Coulombic interaction, $-\mu_{0} q_{m}^{2} /(4 \pi r)$, mediated by monopolar magnetic fields, see Fig. 3 .

This interaction is genuinely magnetic, hence the presence of the vacuum permeability $\mu_{0}$, and not $1 / \epsilon_{0}$. It takes only a finite energy to separate the monopoles to infinity (i.e., they are deconfined), and thus they are the true elementary excitations of the system: the local dipolar excitation fractionalizes!

The reader may now feel that we have slipped the baby in with the bathwater - that in taking the pictures from the dumbbell representation seriously we are somehow introducing monopoles where there were none to begin with. In general, it is of course well known that a string of dipoles arranged head to tail realises a monopole-antimonopole pair at its ends 18 . However, in order to obtain deconfined monopoles, it is essential that the cost of creating such a string of dipoles remain bounded as its length grows, i.e., that the relevant string tension vanish. This is evidently not true in a vacuum (such as that of the universe) where the growth of the string can only come at the cost of creating additional dipoles. Magnetic materials, which come equipped with vacua (ground states) filled with magnetic dipoles, are more promising. However, even here a dipole string is not always a natural excitation, and when it is - e.g., in an ordered ferromagnet - a string of inverted dipoles is accompanied by costly domain walls along 


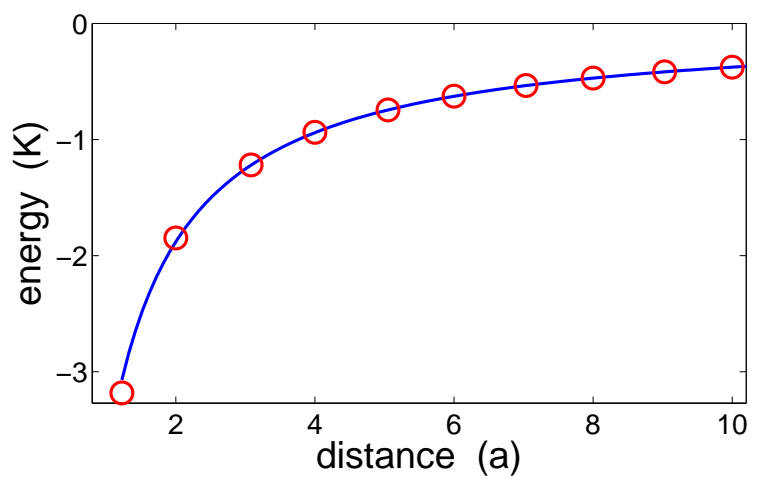

FIG. 3: Monopole interaction. Comparison of the magnetic Coulomb energy $-\mu_{0} q_{m}^{2} /(4 \pi r)$ (Eq. 2] solid line) with a direct numerical evaluation of the monopole interaction energy in dipolar spin ice (Eq. 1 open circles), for a given spin-ice configuration (Fig. 2), as a function of monopole separation.

its length (except, as usual, for one-dimensional systems ${ }^{19}$ ), causing the incipient monopoles to remain confined.

The magic of spin ice arises from its exotic ground states. The ice rule can be viewed as requiring that two dipole strings enter and exit each site of the diamond lattice. In a typical spin ice ground state, there is a "soup" of such strings: many dipole strings - of arbitrary size and shape - can be identified which connect a given pair of sites. Inverting the dipoles along any one such string creates a monopole-antimonopole pair on the sites at its ends. The associated energy cost does not diverge with the length of the string, unlike the case of an ordered ferromagnet, as no domain walls are created along the string, and the monopoles are thus deconfined.

Notice that we did not make reference to the famous Dirac condition $\frac{17}{}$ that the fundamental electric charge $e$ and any magnetic charge $q$ must exhibit the relationship $e q=n h / \mu_{0}$ whence any monopoles in our universe must be quantised in units of $q_{D}=h / \mu_{0} e$. This follows from the monopole being attached to a Dirac string which has to be unobservable ${ }^{18}$ By contrast, the string soup characteristic of spin ice at low temperature makes the strings energetically unimportant, although they are observable and thus not quantised.

Indeed, the monopoles in spin ice have a magnitude

$$
\begin{aligned}
q_{m} & =\frac{2 \mu}{a_{d}} \\
& =\frac{2 \mu}{\mu_{b}} \frac{\alpha \lambda_{C}}{2 \pi a_{d}} q_{D} \\
& \approx \frac{q_{D}}{8000},
\end{aligned}
$$

where $\lambda_{C}$ is the Compton wavelength for an electron, and $\alpha$ is the fine-structure constant. Amusingly, the charge of a monopole in spin ice can even be tuned continuously by applying pressure, as this changes the value of $\mu / a_{d}$.

The monopoles are sources and sinks of the magnetic field $\mathbf{H}$, as is appropriate to the condensed matter setting. More precisely, like in other instances of fractionalisation ${ }^{20}$, one can define a 'smeared' magnetic charge

$$
\rho_{m}(\mathbf{R})=\int d^{3} \mathbf{r}^{\prime} e^{-\left|\mathbf{r}^{\prime}-\mathbf{R}\right|^{2} / \xi^{2}} \nabla \cdot \mathbf{H},
$$

where $\nabla \cdot \mathbf{H}$ is the divergence of the magnetic field. For a monopole at the origin, separated by $L \gg \xi \gg a$ from any other monopoles, this gives $\rho_{m}(0)= \pm q_{m}$. The form of the magnetic induction $\mathbf{B}$ is also monopolar, but with the important difference that a compensating flux travels along the (unquantised) "Dirac string" of flipped dipoles created along with the monopole (see Fig. 2).

Our magnetic monopoles would in principle show up in one of the best-known monopole searches, the Stanford experiment to detect fundamental magnetic monopoles from cosmic radiation. This experiment is based on the fact that a longlived current is induced in a superconducting ring when a monopole passes through it ${ }^{8}$. One can easily check that the presence of the Dirac string of flipped dipoles is immaterial to the establishment of a current.

The above observations are the central qualitative results of our work: ice-rule-violating defects are deconfined monopoles of $\mathbf{H}$, they exhibit a genuine magnetic Coulomb interaction, Eq. 2, and they produce Faraday electromotive forces in the same way as elementary monopoles would.

We reemphasize that the ice rule alone does not permit a consistent treatment of the excited states of the physical problem: crucially, the energetic interaction between our defects is absent altogether. Also, in previous discussions of the purely ice-rule problem and related short ranged problems $\frac{11,12,13}{1 t}$ has been noted that the defects do acquire a purely entropic Coulombic (i.e., $1 / r$ ) interaction that has a strength that vanishes proportionally to $T$ at low temperatures. This interaction will be present in addition to the magnetic Coulomb interaction discussed in this paper, and is clearly much smaller as $T \rightarrow 0$. Also, it will not be accompanied by a magnetic field, it will not renormalise the monopole charge, and it will not be felt by a stationary magnetic test particle embedded in the lattice but not attached to a lattice site.

The most satisfactory way to demonstrate the presence of a monopole would be to measure the force on magnetic test particles, say by a Rutherford scattering experiment or by clever nanotechnological means. Unfortunately, for lack of elementary magnetic monopoles, one would have to choose dipoles as test particles, which significantly weakens such signatures.

An alternative strategy is to look for consequences of the presence of magnetic monopoles in the collective behaviour of spin ice. This is most elegantly achieved by applying a magnetic field in the [111] crystallographic direction. Such field acts as a (staggered) chemical potential, see Fig. 2, favouring the creation of monopoles of a given sign on either sublattice of the diamond lattice.

We thus have a tuneable lattice gas of magnetic monopoles on the diamond lattice. The basic structure of the phase diagram as a function of magnetic field and temperature can be inferred from a series of papers by M. E. Fisher and collaborators 21 in the context of ionic lattice gases and Coulombic criticality. At high $T$, there is no phase transition but a continuous crossover between the high- and low-density 
phases as the chemical potential is varied. At low $T$, a firstorder phase transition separates the two regimes. This transition terminates in a critical point at $\left(h_{c}, T_{c}\right)$, not unlike the liquid-gas transition of water. This serves as a useful diagnostic as the liquid-gas transition is absent for a nearest-neighbour spin-ice model, in which defects interact only entropically. In that case, it is known that there cannot be a first-order transition in the limit of low $T^{22}$.

To confirm this scenario, we have demonstrated by Monte Carlo simulations that the actual phase diagram of dipolar spin ice model has precisely this structure. In order to rule out the appearance of the liquid-gas transition due to effects introduced by the approximations leading to Eq. 2, we simulated directly the original dipolar spin ice model, Eq. 1. The resulting phase diagram is depicted in Fig. 4. The critical endpoint is located around $\left(T_{c}, h_{c}\right)=(0.57 \pm 0.06 \mathrm{~K}, 0.86 \pm 0.03 \mathrm{~T})$. The error bars are mainly due to finite-size effects, as the intensive nature of the simulations of long-range dipolar interactions prohibits simulating very large systems.

This scenario is indeed observed experimentally in spin-ice materials 6.7 , and our results provide a natural explanation. Spin ice in a [111] magnetic field is a very interesting problem which has already attracted considerable attention. The low-density phase of monopoles is known as kagome ice, a quasi two-dimensional phase with algebraic correlations and a reduced residual entropy $6,7,23$. The high-density phase is an ordered state with maximal polarisation along the field direction. Experimental results on the liquid-gas transition and its endpoint are also displayed in Fig. 4 for comparison. Our numerical results are in good qualitative agreement with both experiment and the analytic calculations of Ref.21. Our value of the critical field agrees with Ref. 6 within a few percent, less than the uncertainty due to demagnetisation effects 6,7 . However, the experimental value of $T_{c}$ is about a third lower than the numerical one, most likely due to farther-neighbour (exchange) interaction terms, which despite being small can shift the location of a transition temperature considerably ${ }^{10}$.

The presence of a liquid-gas transition was noted to be very remarkable as there are few, if any, other experimentally known instances in localised spin systems ${ }^{6}$. No mechanism was known to account for this phenomenon, and our theory of magnetic monopoles fills this gap.

The existence of magnetic monopoles in a condensed matter system is, given their intellectual pedigree, exciting in itself. (The reader familiar with the beautiful work on the anomalous Hall effect will notice that the monopoles appearing there are not excitations and do not involve the physical magnetic field ${ }^{24}$.) This is further heightened by their being a rare instance of high-dimensional fractionalisation, a phenomenon of great interest in fields as diverse as correlated electrons and topological quantum computing 25 . We hope our analysis will encourage our experimentalist colleagues to try their hand at directly detecting these monopole. There are surely many avenues to explore in search of useful signatures: scattering, transport and noise measurements, flux detection, to mention just a few.

\section{Acknowledgments}

The authors would like to thank S. Bramwell, J. Chalker, C. Chamon and S. Kivelson (especially for pointing out Ref. 8) for discussions. This work is supported in part by EPSRC Grant No. GR/R83712/01 (CC), and NSF Grant No. DMR 0213706 (SLS). The authors are deeply indebted to A. Canossa for support with the graphics.

\section{APPENDIX A: THE DUMBELL PICTURE}

This material presents a detailed derivation of the dumbell Hamiltonian used extensively in our paper. We start from the generally accepted Hamiltonian which contains a sum of nearest-neighbour exchange and long range dipolar interactions,

$$
\begin{aligned}
H & =\frac{J}{3} \sum_{\langle i j\rangle} S_{i} S_{j} \\
& +D a^{3} \sum_{(i j)}\left[\frac{\hat{e}_{i} \cdot \hat{e}_{j}}{\left|\mathbf{r}_{i j}\right|^{3}}-\frac{3\left(\hat{e}_{i} \cdot \mathbf{r}_{i j}\right)\left(\hat{e}_{j} \cdot \mathbf{r}_{i j}\right)}{\left|\mathbf{r}_{i j}\right|^{5}}\right] S_{i} S_{j} .
\end{aligned}
$$

The magnetic moment of a spin is denoted by $\mu$, which equals approximately 10 Bohr magnetons $\left(\mu=10 \mu_{B}\right)$ for the spin ice compounds discussed here (namely, $\mathrm{Dy}_{2} \mathrm{Ti}_{2} \mathrm{O}_{7}$ and $\left.\mathrm{Ho}_{2} \mathrm{Ti}_{2} \mathrm{O}_{7}\right)$. The distance between spins is $r_{i j}$, and $a \simeq 3.54 \AA$ is the pyrochlore nearest-neighbour distance. $D=\mu_{0} \mu^{2} /\left(4 \pi a^{3}\right)=1.41 \mathrm{~K}$ is the coupling constant of the dipolar interaction.

A dipole can be thought of as a pair of equal and opposite charges of strength $\pm q$, separated by a distance $\tilde{a}$, such that $\mu=q \tilde{a}$. The dipolar part of the Hamiltonian is reproduced exactly in the limit $\tilde{a} \rightarrow 0$. Here we choose $\tilde{a}$ to equal the diamond lattice constant $a_{d}=\sqrt{3 / 2} a$, therefore fixing $q=$ $\mu / a_{d}$. The two ways of assigning charges reproduce the two orientations of the original dipole, as illustrated in Fig.5.

Given a configuration of $N$ dipoles, let us label $\left\{q_{i}, i=\right.$ $1, \ldots, 2 N\}$ the $2 N$ charges in the corresponding dumbell configuration. The magnetic Coulomb interaction between the charges is given by

$$
\mathcal{V}\left(r_{i j}\right)= \begin{cases}\frac{\mu_{0}}{4 \pi} \frac{q_{i} q_{j}}{r_{i j}} & r_{i j} \neq 0 \\ v_{0} q_{i} q_{j} & r_{i j}=0,\end{cases}
$$

which reproduces the interaction between two dipoles exactly in the limit of large separation between them (modulo an innocuous "self-energy" term discussed below). We can also tune the onsite contribution $v_{0}$ so as to match the interaction energy $\pm J_{\text {eff }}= \pm(J+5 D) / 3$ between two neighbouring dipoles on the lattice (see for example Fig. 6). For two neighbouring spins both pointing into the shared tetrahedron (right panel of Fig. (6) one has

$$
\begin{aligned}
J_{\text {eff }} & =v(0)-2 v\left(r_{12}\right)-2 v\left(r_{23}\right)+v\left(r_{13}\right) \\
& =v(0)-v\left(r_{12}\right)-v\left(r_{23}\right)+2 E_{\text {self }}+v\left(r_{13}\right),
\end{aligned}
$$




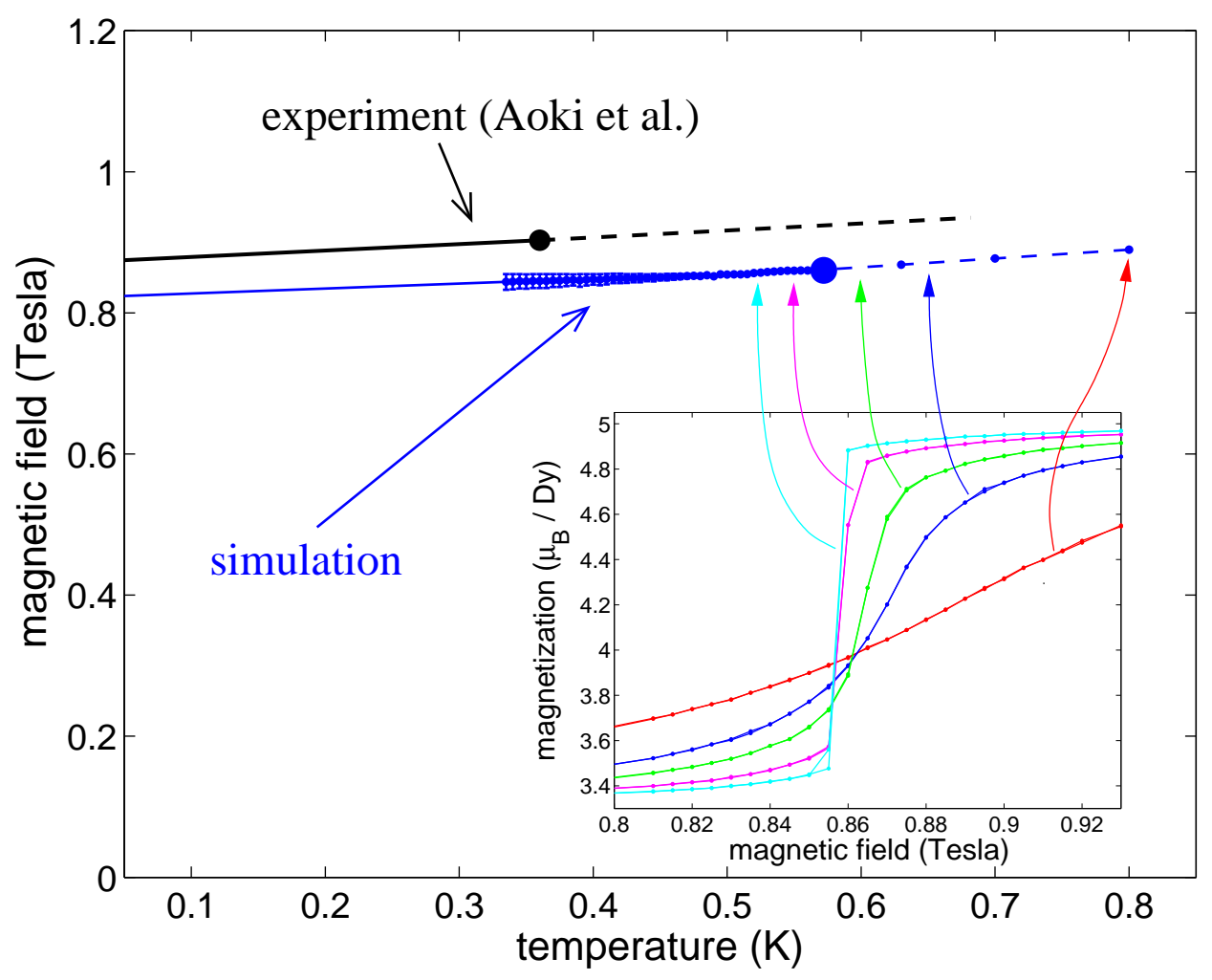

FIG. 4: Phase diagram of spin ice in a [111] field. The location of the monopole liquid-gas transition from numerics (blue line) compared to experiment (black line). The solid line is a first-order transition terminated by a critical endpoint (fat circle). The dashed lines are crossovers. Inset: magnetisation curves showing the onset of first-order behaviour as the temperature is lowered. Our simulations cover the range $0.335 \mathrm{~K}<T<0.8 \mathrm{~K}$ for 1024 spins. At the lowest temperatures, the parallel tempering code we use in our simulations of the Ewald-summed dipolar interaction no longer completely suppresses the hysteresis, and we have extended the first-order transition line using Clausius-Clapeyron.

where $v(r)=|\mathcal{V}(r)|$, and $E_{\text {self }}=-v\left(r_{12}\right)=-v\left(r_{23}\right)$ is the self-energy of a dipole in the dumbell picture. For two neighbouring spins pointing into and out of the shared tetrahedron
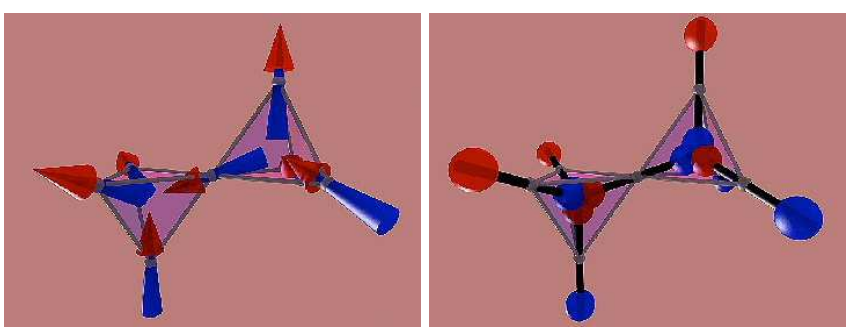

FIG. 5: Mapping from dipoles to dumbells. Left: two neighboring tetrahedra obeying the ice rule, with two spins pointing in and two out, giving zero net charge on each site. Right: The corresponding dumbell picture obtained by replacing each spin by a pair of opposite magnetic charges placed on the adjacent sites of the diamond lattice.

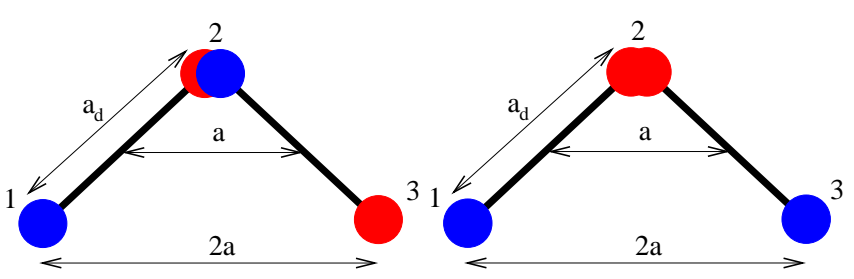

FIG. 6: Schematic examples of two neighbouring dipoles on the pyrochlore lattice.

(as in the left panel of Fig. 6), we obtain

$$
\begin{aligned}
-J_{\mathrm{eff}} & =-v(0)+2 v\left(r_{12}\right)+2 v\left(r_{23}\right)-v\left(r_{13}\right) \\
& =-v(0)+v\left(r_{12}\right)+v\left(r_{23}\right)+2 E_{\mathrm{self}}-v\left(r_{13}\right) .
\end{aligned}
$$

Taking the difference between the two equations above, and using the fact that $r_{12}=r_{23}$ and therefore $v\left(r_{12}\right)=v\left(r_{23}\right)$, one gets

$$
v(0)=J_{\text {eff }}+2 v\left(r_{12}\right)-v\left(r_{13}\right)
$$


where

$$
\begin{aligned}
r_{12} & =a_{d} \\
r_{13} & =2 a \\
v(r) & = \begin{cases}\frac{\mu_{0}}{4 \pi} \frac{\left(\mu / a_{d}\right)^{2}}{r} & r \neq 0 \\
v_{0}\left(\frac{\mu}{a_{d}}\right)^{2} & r=0\end{cases}
\end{aligned}
$$

and in the end

$$
v(0)=\frac{J+5 D}{3}+\frac{\mu_{0}}{4 \pi}\left(\frac{\mu}{a_{d}}\right)^{2}\left[\frac{2}{a_{d}}-\frac{1}{2 a}\right]
$$

Using the fact that $D=\mu_{0} \mu^{2} / 4 \pi a^{3}$ and that $a=a_{d} \sqrt{2 / 3}$, the expression above reduces to

$$
v_{0}\left(\frac{\mu}{a_{d}}\right)^{2}=\frac{J}{3}+\frac{4}{3}\left[1+\sqrt{\frac{2}{3}}\right] D .
$$

Now, the energy of a charge configuration can be straightforwardly resummed and written in terms of the total charges
$Q_{\alpha}$ on each site $\alpha$ of the diamond lattice, i.e., $Q_{\alpha}=q_{i_{1}}+$ $q_{i_{2}}+q_{i_{3}}+q_{i_{4}}$ for the quartet of charges with $r_{i_{1}}=r_{i_{2}}=$ $r_{i_{3}}=r_{i_{4}} \equiv r_{\alpha}$. The energy can thus be computed from a pairwise Coulomb interaction of the magnetic charges $Q_{\alpha}$ :

$$
V\left(r_{\alpha \beta}\right)= \begin{cases}\frac{\mu_{0}}{4 \pi} \frac{Q_{\alpha} Q_{\beta}}{r_{\alpha \beta}} & \alpha \neq \beta \\ \frac{1}{2} v_{0} Q_{\alpha}^{2} & \alpha=\beta,\end{cases}
$$

This gives the same total energy as Eq. A2 up to an unimportant overall constant $(1 / 2) \sum q_{i}^{2}=N\left(\mu / a_{d}\right)^{2}$. Thus, it is also equivalent to the dipolar energy Eq. A1 up to corrections which are small everywhere, and vanish with distance at least as fast as $1 / r^{5}$ for each dipole pair. In particular, one can show that $v_{0}$ is large enough, for the compounds we consider, that it enforces $Q_{\alpha}=0$ everywhere in the ground state. These are just the ice rules, and the degeneracy of all the states follows from the fact that all terms with $r_{\alpha \beta}>0$ vanish for $Q_{\alpha} \equiv 0$.
1 See, e.g., Milton, K. A. Rep. Progr. Phys. 69, 1637-1712 (2006).

${ }^{2}$ Laughlin, R. B., Nobel Lecture: Fractional quantization, Rev. Mod. Phys. 71, 863-874 (1999).

${ }^{3}$ Harris, M. J., Bramwell, S. T., McMorrow, D. F., Zeiske, T., and Godfrey, K. W., Geometrical frustration in the ferromagnetic pyrochlore $\mathrm{Ho}_{2} \mathrm{Ti}_{2} \mathrm{O}_{7}$, Phys. Rev. Lett. 79, 2554-2557 (1997).

${ }^{4}$ For a review of spin ice, see Bramwell, S. T., and Gingras, M. J. P., Spin ice state in frustrated magnetic pyrochlore materials. Science 294, 1495-1501 (2001).

5 Ramirez, A. P., Hayashi, A., Cava, R. J., Siddharthan, R. B., and Shastry, S., Zero-point entropy in 'spin ice'. Nature 399, 333-336 (1999).

6 Aoki, H., Sakakibara, T., Matsuhira, K., and Hiroi, Z., Magnetocaloric effect study on the pyrochlore spin ice compound $\mathrm{Dy}_{2} \mathrm{Ti}_{2} \mathrm{O}_{7}$ in a [111] magnetic field. J. Phys. Soc. Jpn. 73, 28512856 (2004).

7 Higashinaka, R., Fukazawa, H., Deguchi, K., and Maeno, Y., Low temperature specific heat of $\mathrm{Dy}_{2} \mathrm{Ti}_{2} \mathrm{O}_{7}$ in the kagome ice state. J. Phys. Soc. Jpn. 73, 2845-2850 (2004).

${ }^{8}$ Cabrera, B., First results from a superconductive detector for moving magnetic monopoles. Phys. Rev. Lett. 48, 1378-1381 (1982).

9 Siddharthan, R. et al., Ising Pyrochlore Magnets: LowTemperature Properties, Ice Rules, and Beyond, Phys. Rev. Lett. 83, 1854-1857 (1999).

${ }^{10}$ Ruff, J. P. C., Melko, R. G, and Gingras, M. J. P., Finitetemperature transitions in dipolar spin ice in a large magnetic field. Phys. Rev. Lett. 95, 097202 (2005).

11 Huse, D. A., Krauth, W., Moessner, R., and Sondhi, S. L., Coulomb and liquid dimer models in three dimensions. Phys. Rev. Lett. 91, 167004-4 (2003).

${ }^{12}$ Hermele, M., Fisher, M. P. A., Balents, L., Pyrochlore photons:
The $\mathrm{U}(1)$ spin liquid in a $\mathrm{S}=1 / 2$ three-dimensional frustrated magnet. Phys. Rev. B 69, 064404-21 (2004).

13 Henley, C. L., Power-law spin correlations in pyrochlore antiferromagnets. Phys. Rev. B 71, 014424-11 (2005).

14 Fulde, P., Penc, K., and Shannon, N., Fractional charges in pyrochlore lattices. Ann. Phys. 11, 892-900 (2002).

15 Levin, M., and Wen, X. G., Quantum ether: Photons and electrons from a rotor model, Phys. Rev. B 73, 035122-10 (2006).

${ }^{16}$ Nagle, J. F., Theory of the dielectric constant of ice. Chem. Phys. 43, 317-328 (1979).

17 Dirac, P. A. M., Quantised singularities in the electromagnetic field. Proc. Roy. Soc. A 133, 60 (1931).

18 Jackson, J. D., Classical Electrodynamics (Wiley, New York, 1975).

19 Saitoh, E., Miyajima, H., Yamaoka, T., and Tatara,G., Currentinduced resonance and mass determination of a single magnetic domain wall. Nature 432, 203-206 (2004).

${ }^{20}$ Rajaraman, R., Fractional charge. http://arxiv.org/abs/condmat/0103366v1 (2001).

${ }^{21}$ Kobelev, V., Kolomeisky, A. B., and Fisher, M. E., Lattice models of ionic systems. J. Chem. Phys. 116, 7589-7598 (2002).

${ }^{22}$ Heilmann, O. J., and, Lieb, E. H., Theory of monomer-dimer systems. Comm. Math. Phys. 25, 190-232 (1972).

23 Isakov, S. V., Raman, K. S., Moessner, R., and Sondhi, S. L., Magnetization curve of spin ice in a [111] magnetic field. Phys. Rev. B 70, 104418-12 (2004).

${ }^{24}$ Fang, Z. et al., The anomalous Hall effect and magnetic monopoles in momentum space. Science 302, 92-95 (2003).

25 Kitaev, A. Yu., Fault tolerant quantum computing. Ann. Phys. 303, 2-30 (2003). 


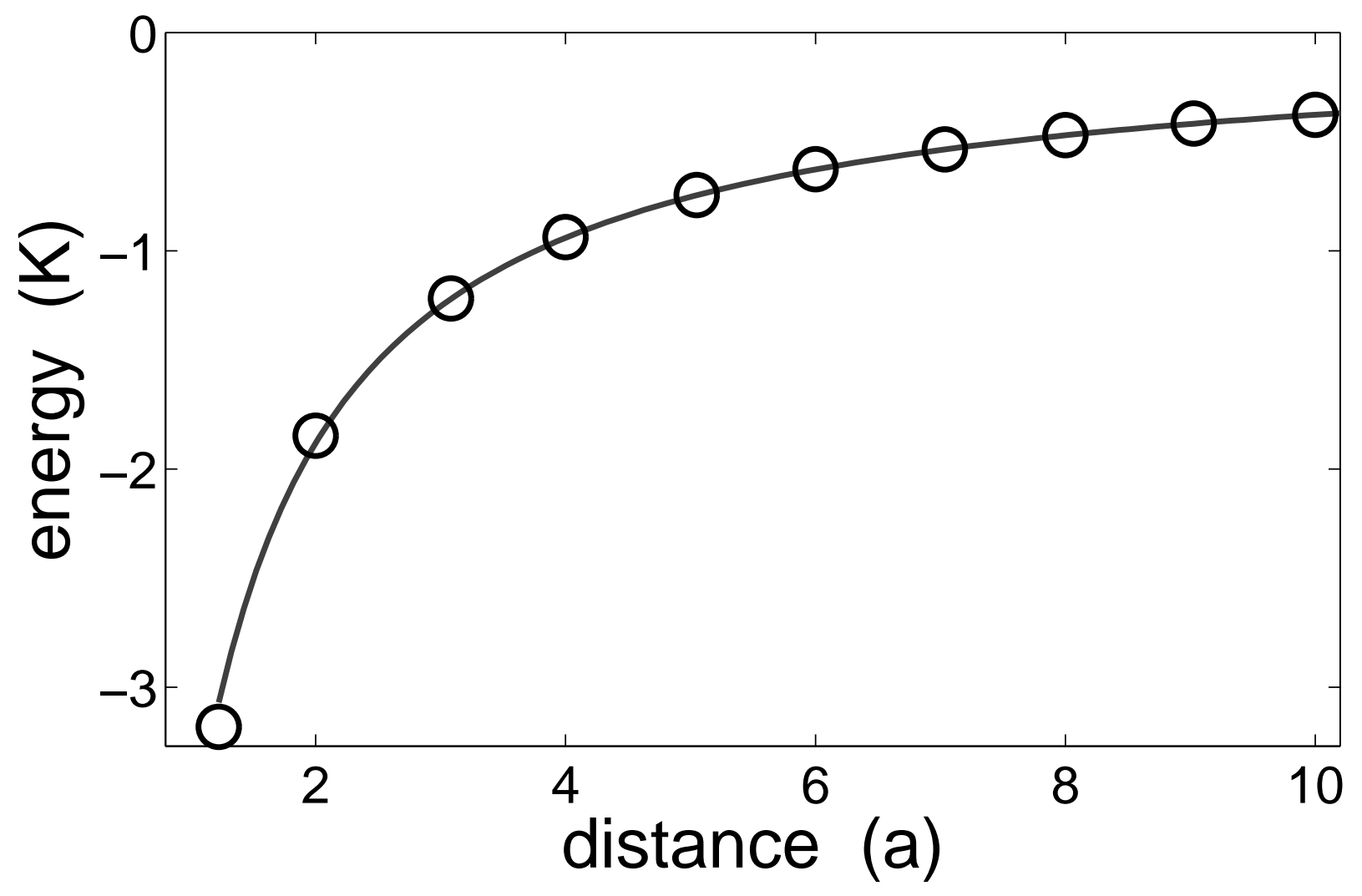

